EPJ Web of Conferences 31, 00036 (2012)

DOI: $10.1051 /$ epjconf/20123100036

(C) Owned by the authors, published by EDP Sciences - SIF, 2012

\title{
Use of fragmentation beams at LNS with CHIMERA detector
}

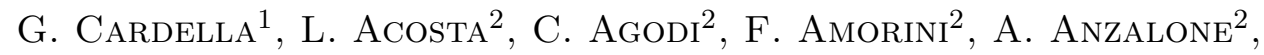
L. Auditore ${ }^{4}$, I. Berceanu ${ }^{7}$, M. Buscemi ${ }^{2,3}$, M. B. Chatterjiee ${ }^{8}$, E. DeFilippo ${ }^{1}$, L. Francalanza ${ }^{2,3}$, R. Gianí ${ }^{2,3}$, L. Grassi ${ }^{1}$, E. La Guidara ${ }^{1}$, G. LAnZalone ${ }^{2,5}$, I. Lombardo ${ }^{6}$, D. Loria ${ }^{4}$, T. Minnitit $^{4}$, A. Pagano ${ }^{1}$, E. V. Pagano ${ }^{2,3}$, M. Papa $^{1}$, S. Pirrone $^{1}$, G. Politi ${ }^{1,3}$, F. Porto ${ }^{2,3}$, F. Rizzo ${ }^{2,3}$, E. Rosato $^{6}$, P. Russotto ${ }^{2,3}$, S. Santoro ${ }^{4}$, A. Trifiró ${ }^{4}$, M. Trimarchi ${ }^{4}$, G. Verde $^{1}$ and M. Vigilante ${ }^{6}$

${ }^{1}$ INFN, Sezione di Catania, Italy

${ }^{2}$ LNS-INFN, Catania, Italy

3 Dipartimento di Fisica e Astronomia Università di Catania, Italy

${ }^{4}$ INFN gr. coll. di Messina e Dip. di Fisica Università di Messina, Italy

${ }^{5}$ Facoltà di Ingegneria e Architettura, Università Kore, Enna, Italy

${ }^{6}$ INFN Sezione di Napoli e Dip. di Fisica Università di Napoli Federico II, Italy

7 National Institute for Physics and Nuclear Engineering Bucharest-Măgurele, Romania

8 Saha Institute for Nuclear Physics, Kolkata, India

\begin{abstract}
The recent intensity upgrade of the LNS fragmentation beam is discussed. The available beams, the tagging procedures and details on the beam quality are reported. The experimental program started with the CHIMERA detector using such beams is also discussed with preliminary results and future perspectives.
\end{abstract}

This is an Open Access article distributed under the terms of the Creative Commons Attribution License 2.0, which permits unrestricted use, distribution, and reproduction in any medium, provided the original work is properly cited. 


\section{Introduction}

After the recent intensity upgrade [1] of the LNS fragmentation beam, relatively large yields of various neutron rich and poor exotic beams, from 20 to $50 \mathrm{MeV} / \mathrm{A}$, are available. For the best use of such beams during 2007-2008 the CHIMERA detector [2] was moved to its new scattering chamber where a better transmission was measured. A tagging system [3] was developed in order to event by event identify the characteristics of the beam particles. Such system was further implemented in order to measure also the beam trajectory. Using these beams, an ambitious experimental program was started based on the study of both structure and dynamical effects on nuclear reactions mechanisms. Measurements on elastic scattering and neutron transfer reactions, on proton and deuteron targets, have been performed. Beams as ${ }^{10,11} \mathrm{Be},{ }^{13} \mathrm{~B},{ }^{16} \mathrm{C}[4]$ produced by fragmentation of ${ }^{13} \mathrm{C}$ and ${ }^{18} \mathrm{O}$ at 55 $\mathrm{MeV} / \mathrm{A}$ were used. Preliminary tests with neutron rich/poor beams in the mass 30-40 range impinging on ${ }^{27} \mathrm{Al}$ targets have also been performed. Such beams were produced by ${ }^{36} \mathrm{Ar}$ fragmentation in order to study the isospin dependence of reaction mechanisms competition at $25 \mathrm{MeV} / \mathrm{A}$ [5]. New tests experiments are in program in the next future on ${ }^{8} \mathrm{He}$ and ${ }^{68} \mathrm{Ni}$ production. In particular ${ }^{68} \mathrm{Ni}$ can be used to extend previous timescale measurements performed with various $\mathrm{Ni}$ and $\mathrm{Sn}$ isotopes [6].

\section{Beam production, selection and transport}

The beam is produced mainly by fragmentation reactions, using the beams accelerated by the superconducting Cyclotron (CS) of the Laboratori Nazionali del Sud in Catania. A Beryllium target is placed near the CS between two triplets of quadrupole. The quadrupole setting is optimized in order to produce the smallest possible beam spot on the target and to collect the maximum yield for the reaction fragments. The standard primary beam intensity up to now used is of the order of 100 Watts. On average $20 \%$ of the beam energy is lost in the production target. The target must be therefore cooled and can be also continuously moved, during the experiment, in order to better distribute the heat for a safe operation. The beam selection is obtained with appropriate setting of the first two $45^{\circ}$ dipoles of the main beam transport line of the CS. In the middle of the two dipoles there is the possibility to insert a wedge to improve the quality of the beam selection, with the cost of intensity reduction.

The beam transport has also been recently very much improved, due to 


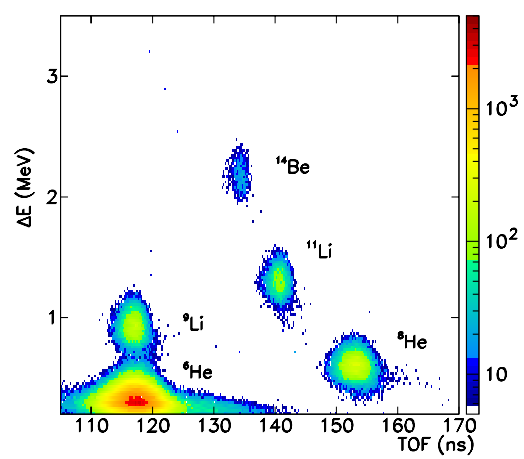

Figure 1: (colour online) Identification scatter plot for the production of ${ }^{11} \mathrm{Li} /{ }^{8} \mathrm{He}$ beams.

the installation of new diagnostic devices [7], originally developed for the EXCYT beam [8]. About 11 diagnostic points were installed, up to the CHIMERA scattering chamber, each one equipped with a $\mathrm{x}-\mathrm{y}$ position sensitive silicon detector and a plastic scintillator. The plastic scintillator is used, as a Faraday cup, to count the beam particles, while the silicon detector can show the shape of the beam envelop, as an alumina. Both diagnostic elements can give the energy loss spectrum, and can be in principle used for the beam identification when also the time information is used.

\section{The tagging method}

The tagging system is an evolution of the first tagging system used for fragmentation experiment at LNS [9]. It is based on the use of a double side silicon strip detector (DSSSD) measuring the energy loss of the impinging beam particles. The identification of the particles is achieved combining this energy loss with the time of flight information (TOF) of the particle. The start of this TOF measurement is provided by a large size micro-channel plate (MCP) mounted $13 \mathrm{~m}$ away from the DSSSD. The stop is given by the front strips of the DSSSD. The use of the MCP [3] in the TOF measurement allows to overcome the $30 \mathrm{~ns}$ maximum range limitation given by the cycle of the CS radiofrequency. An example of the obtained identification scatter plot is shown in fig. 1 for the production of ${ }^{11} \mathrm{Li}$ by ${ }^{18} \mathrm{O}$ fragmentation. Exotic beams as ${ }^{8} \mathrm{He}$ and ${ }^{14} \mathrm{Be}$ are also observed. We have tested the performance of the MCP up to a counting rate of the order of $1 \mathrm{MHz}$. 

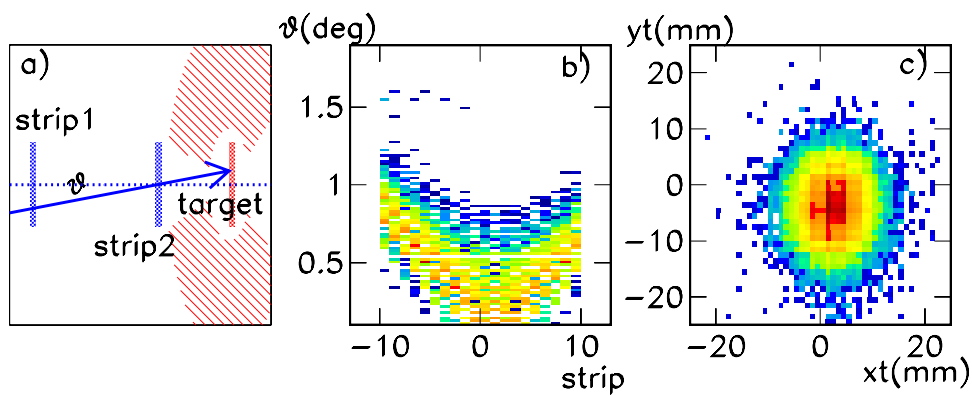

Figure 2: (colour online) a) Graph not in scale of the trajectory measurement system. b) $\theta$ (deg) beam impinging angle as a function of the horizontal strip position on the tagging DSSSD. c) shape of the fragmentation beam on the target.

We measured an overall time resolution better than 500 ps from one pixel of the DSSSD detector and the MCP. The DSSSD provides also the position information. This is however not enough to precisely determine the beam trajectory. To measure trajectories we have placed, at the entrance of the CHIMERA sphere, another DSSSD detector mounted on a remotely movable arm. The position information provided by both DSSSD allows to extract the beam trajectory, and it is also very useful to sketch the beam profile at the target position. In fig. 2a we show a draw not in scale of the set-up for the trajectory measurement. The first DSSSD detector is placed around $2 \mathrm{~m}$ away from the target, the second one $70 \mathrm{~cm}$ before the target. In this figure we indicate as $\theta$, the beam impinging angle. In fig. $2 \mathrm{~b}$ the plot of $\theta$ as a function of the horizontal strip number of the first DSSSD is shown. A rather clear correlation can be observed. From this picture we note that the fragmentation beam can be focused with a relatively small divergence with a maximum impinging angle $\theta$ of about $1^{\circ}$. Moreover for a fixed horizontal strip we have a relatively narrow distribution of $\theta$ with a spread of the order of $\pm 0.2^{o}$ Therefore the event by event trajectory measurement is necessary only if the required angular resolution is better than $0.5^{\circ}$. In the other cases the impinging angle can be more simply reconstructed by the $\mathrm{x}$ coordinate of the beam on the tagging DSSSD. From the trajectory measurement one can reconstruct the beam shape on the target shown in fig $2 \mathrm{c}$. We note that it is possible to focus the beam on a spot with a diameter of the order of 2 $\mathrm{cm}$. 


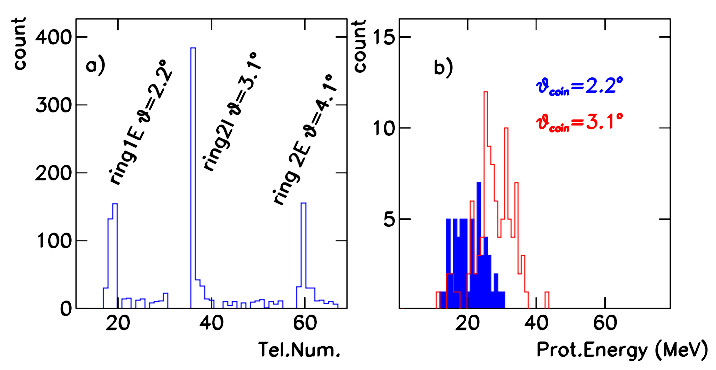

Figure 3: (colour online) a) Coincidences of forward detectors with a telescope at $66^{\circ}$. b) Proton energy spectra detected at $66^{\circ}$ in coincidence with ${ }^{16} \mathrm{C}$ elastically scattered at $2.2^{\circ}$ (filled spectrum) and $3.1^{\circ}$.

\section{Preliminary results}

As described in the introduction we have performed various test experiments. A first class of experiments concerns the study of elastic scattering and transfer reactions of neutron rich fragments on proton and deuteron targets. For such experiments we exploited the kinematic coincidence technique [10] in order to clean from the background the reaction cross section. The heavy projectile like fragment is detected in the very forward CHIMERA rings, from $1^{o}$ to $10^{\circ}$, depending on the kinematics of the reactions, while the light partner is detected up to $90^{\circ}$ for elastic scattering on proton targets or on the whole detector in case of $(d, p)$ reaction. A kinematic coincidence can be observed only from detectors with a difference in the azimuthal angle $\phi$ of $180^{\circ}$. This constraint strongly reduces the contamination due for instance to reactions with the carbon of the plastic target used. The amount of the cleaning efficiency can be evaluated by looking at fig. 3a where the coincidences from a telescope placed at $66^{\circ}$ with telescopes of the forward rings are plotted as a function of the telescope number. We see some peaks corresponding to telescopes of the various rings placed at the right $\phi$ angle satisfying the rule $\Delta \phi=180^{\circ}$.

Selecting the desired beam and the right kinematics we can finally get the energy spectra of detected particles in various kind of reactions. In fig. $3 \mathrm{~b}$ we can see, for instance, the energy plot of protons detected at $66^{\circ}$. The two spectra shown are collected in coincidence with ${ }^{16} \mathrm{C}$ elastically scattered at two different angles $2.2^{\circ}$ (filled spectrum) and $3.1^{\circ}$. We note the shift in proton energy due to the kinematics of the reaction. The two spectra have an overlap due to the fact that the detection angle should be 


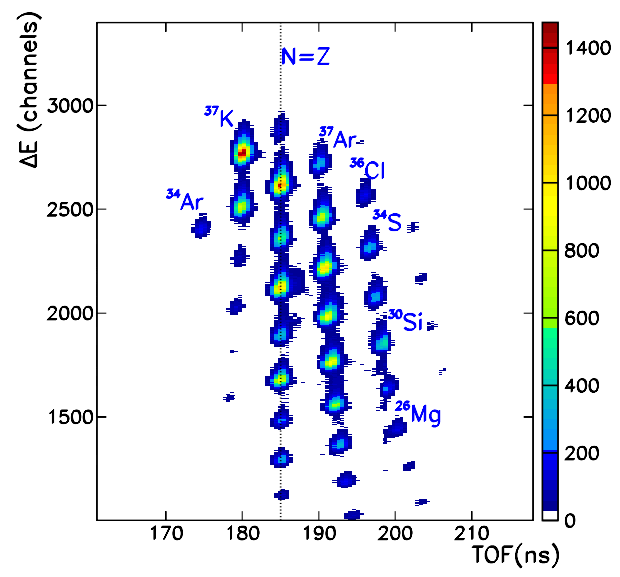

Figure 4: (colour online) Identification scatter plot of fragmentation beams produced with ${ }^{36} \mathrm{Ar}$ primary beam. The $\mathrm{N}=\mathrm{Z}$ line is evidenced. Nuclei with the same $\mathrm{N} / \mathrm{Z}$ must have the same velocity due to $\mathrm{B} \rho$ selections.

corrected for the beam impinging angle $\theta$ shown in fig. 2a,b. The width of the proton energy distribution is partially due to kinematics broadening due to the finite opening angle of our detectors $\left( \pm 4^{\circ}\right.$ for the detector at $\left.66^{\circ}\right)$. The spread in the beam energy also contribute to this width. The transport beam line has in fact a finite momentum acceptance $\Delta \mathrm{P} / \mathrm{P}=1 \%$. This means that the energy spread of the beam is of the order of $2 \%$. To extract very precise angular distributions or excitation energy spectra also this energy spread must be considered. The beam energy can be measured in various way, using the TOF measurement or using the $\mathrm{B} \rho$ dispersion of the last dipole of the beam line on the tagging DSSSD detector. Work is in progress to optimize such measurements.

\section{$5 \quad$ Future perspectives}

On 2012 other attempts to produce new beams are planned. The first test experiment will be the production of ${ }^{68} \mathrm{Ni}$ beam trough fragmentation of ${ }^{70} \mathrm{Zn}$. A beam intensity of $5 \cdot 10^{4}$ to $10^{5}$ is foreseen by LISE calculations. A primary beam intensity of 100 Watt on a ${ }^{9} \mathrm{Be}$ target of $250 \mu \mathrm{m}$ will be used. This beam will constitute a very challenging proof for the tagging system. We foreseen a total yield rather near to $10^{6}$ part./s, the energy loss 
in the tagging DSSSD detector (140 $\mu \mathrm{m}$ thick) will be more than $300 \mathrm{MeV}$ so radiation damage will be probably an issue. For this measurement we will use a DSSSD detector with 32 strips. The larger number of strips is important to share the yield on the largest possible number of channels so reducing the pile-up and the radiation damage for the single strip. Following the encouraging results obtained with ${ }^{36} \mathrm{Ar}$ primary beam, shown in fig.4, where a rather good particle identification was obtained (200 kHz counting rate on 24 strips) we hope to be able to maintain satisfying results. The main aim of the test is to perform new measurements on reaction on various Sn isotopes in order to follow the isospin dependence of the emission timescale of fragments produced by the neck fragmentation [6].

Another challenging experiment will be pursued once a ${ }^{11} \mathrm{~B}$ beam will be produced by the cyclotron. Such primary beam is in fact the most efficient one in order to produce ${ }^{8} \mathrm{He}$ beam by fragmentation. Lise calculations predict a beam yield of about $2 \mathrm{kHz}$. In order to perform an efficient tagging we will replace the MCP by a plastic scintillator to overcome the low MCP efficiency for such kind of particles. With such beam we can plan interesting experiments to study for instance ${ }^{9} \mathrm{He}$ population and decay (using a deuterated target) or even ${ }^{10} \mathrm{He}$, once a tritium target will be available.

\section{References}

[1] see http://fribs.lns.infn.it/upgrade-results.html

[2] A.Pagano et al Nucl.Phys. A 734 (2004) 504 and ref. therein

[3] I. Lombardo et al Nucl.Phys.B (Proc.Suppl.) 215(2011)272

[4] L.Grassi phd thesis

[5] F.Amorini et al, Phys. Rev. Lett. 102, 112701(2009).

[6] E.De Filippo et al, Phys. Rev. C 71, 044602 (2005).

[7] A.Amato et al Report LNS 2009 pag 160.

[8] G.Cuttone et al NIM B261, 1040(2007).

[9] G.Raciti et al Phys.Rev.Lett. 100, 19250(2008).

[10] G.Cardella et al: Proc. of the Conf. Nuclear structure and dynamics 09 Dubrovnik, pag. 309, American Institute of Physics. 